\title{
The Clinical Effect of Blue Light Therapy on Patients with Delayed Sleep-Wake Phase Disorder
}

\author{
Dong $\mathrm{Li}^{1, *}$ \\ Penghong Fang ${ }^{2, *}$ \\ Huimiao Liu $^{3}$ \\ Lili Chen ${ }^{4}$ \\ Ying $\mathrm{Fu}^{5}$ \\ Junqian Liu $^{5}$ \\ Bingchuan $\mathrm{Xie}^{3}$ \\ Yihan Liu $^{3}$ \\ Hongyuan $\mathrm{Ye}^{6}$ \\ Ping $\mathrm{Gu}^{3}$
}

'Department of Vertigo Center, The First Hospital of Hebei Medical University, Shijiazhuang, 05003I, Hebei, People's Republic of China; ${ }^{2}$ Department of Neurology, Xiangya Changde Hospital, Changde, 4I5000, Hunan, People's Republic of China; ${ }^{3}$ Department of Neurology, The First Hospital of Hebei Medical University, Shijiazhuang, 05003I, Hebei, People's Republic of China; ${ }^{4}$ Department of Neurology, Tangshan Workers' Hospital, Tangshan, 063000, Hebei, People's Republic of China;

${ }^{5}$ Department of Brain Function, The First Hospital of Hebei Medical University, Shijiazhuang, 05003I, Hebei, People's Republic of China; ${ }^{6}$ Department of Neurology, North China University of Science and Technology Affiliated Hospital, Tangshan, 063000, Hebei, People's Republic of China

*These authors contributed equally to this work

Correspondence: Ping Gu Department of Neurology, The First Hospital of Hebei Medical University, No. 89 Donggang Road, Shijiazhuang, 05003I, People's Republic of China Tel +86-18101169132

Email1057076160@qq.com
Objective: To investigate the feasibility and patient acceptance of applying blue light glasses to treat delayed sleep-wake phase disorder (DSWPD).

Methods: Fifteen patients with DSWPD were collected as the observation group and 15 healthy people as the control group. The patients wore blue light glasses with a continuous radiation wavelength of about $470 \mathrm{~nm}$ for $1 \mathrm{~h}$ to $2 \mathrm{~h}$ during the period from 06:30 to 09:00 in the morning after waking up, respectively. Assessment of Hamilton Anxiety Scale 14 items (HAMA14), Hamilton Depression Rating Scale 24 items (HAMD24), Pittsburgh Sleep Quality Index (PSQI), Epworth Sleepiness Scale (ESS), Morningness-Eveningness Questionnaire (MEQ), and Insomnia Severity Index (ISI) scores before and after 1 week of treatment. Pearson correlation was used to analyze the correlation between the efficacy of patients with sleep-wake phase delay disorder and HAMA14, HAMD24, PSQI, ISI, ESS, MEQ, SL (sleep time), TST (total sleep time), TTiB (total time in bed), SQ (sleep quality), TOA (total arousal time), WASO (wake after sleep onset), AAT (average arousal time), and SE (sleep efficiency percent). Multi-factor logistic regression analysis of factors influencing the efficacy of patients with sleep-wake phase delay disorder.

Results: After treatment, PSQI-G scores, number of nighttime awakenings and time of awakening recorded in the sleep diary decreased significantly in the observation group $(\mathrm{P}<0.05)$, and subjective sleep quality and MEQ scores increased $(\mathrm{P}<0.05)$. MEQ score shifted from "moderate night type" to "intermediate type", sleep-wake phase tended to shift forward. The total PSQI score and Pittsburgh Sleep Quality Index Global (PSQI-G) score were significantly lower in the control group after treatment $(\mathrm{P}<0.05)$. By Pearson correlation analysis, the efficacy of patients with sleepwake phase delay disorder was significantly correlated with HAMA14, HAMD24, PSQI, ISI, ESS, MEQ, SL, TST, TTiB, SQ, TOA, WASO, AAT, and SE. Multifactorial logistic regression analysis revealed that the factors influencing the efficacy of patients with sleep-wake phase delay disorder were PSQI, ISI, ESS, MEQ, SL, TST, TTiB, SQ, TOA, WASO, AAT, and SE.

Conclusion: Blue light therapy has a positive effect on improving subjective sleep quality, reducing the number of nocturnal awakenings and the duration of nocturnal awakenings, improving daytime function, and shifting the sleep phase forward in patients with DSWPD. Blue light therapy improves subjective sleep quality and daytime function the following day in normal individuals.

Keywords: blue light, sleep-wake phase delay disorder, DSWPD, sleep quality

\section{Introduction}

Delayed sleep-wake phase disorder (DSWPD) is classified as a circadian sleep disorder in the International Classification of Sleep Disorders. ${ }^{1,2}$ Patients with DSWPD have a significant delay in the main sleep period relative to the sleepwake time required for subjective expectations or social life, have difficulty falling asleep and staying awake, and symptoms last for at least 3 months, along with difficulty falling asleep, difficulty waking up, daytime sleepiness, poor energy, and 
are often comorbid with depression. ${ }^{3}$ DSWPD is prevalent in adolescents and young adults, with a prevalence of $7 \%$ to $16 \%{ }^{4,5}$ Because most patients with DSWPD present with difficulty sleeping, they are often misdiagnosed as insomniacs by clinicians. ${ }^{6}$ Previous data show that about $10 \%$ of recurrent insomnia patients in the sleep clinic are DSWPD. $^{7}$

The main points of treatment for DSWPD are to correct disturbed circadian rhythms, improve sleep quality, increase sleep duration and improve daytime function. The main treatment methods currently include chronotherapy, pharmacotherapy and phototherapy. ${ }^{8,9}$ Among them, chronotherapy and pharmacotherapy are used to regulate circadian rhythms and induce earlier sleep phases through behavioral control or medications, respectively, ${ }^{10}$ but they are difficult to be widely used in clinical practice due to inconsistent treatment criteria, numerous side effects and lack of controlled trials to support their efficacy. ${ }^{11}$ Traditional light therapy mainly applies light box for treatment, the treatment process requires the patient to stay in a limited space for at least 30 minutes, site constraints and poor patient acceptance become the difficulty of widespread clinical application. ${ }^{12}$ Blue light has the strongest inhibitory effect on endogenous melatonin secretion and has the most significant effect on the phase change of biological clock. ${ }^{13}$ It has been found that blue light is effective in regulating circadian rhythms, requiring less time as well as lower light irradiance, and the light intensity from the eyes is 110 LUX when blue light glasses are properly worn. ${ }^{14,15}$ On this basis, we propose a hypothesis that portable blue light glasses may be available to treat DSWPD. To confirm this, DSWPD patients were asked to wear circadian-rhythm-regulating LED blue light glass with a wavelength of $460-480 \mathrm{~nm}$. And we analyzed the records of patients' sleep logs before and after treatment and the results of acceptance.

\section{Materials and Methods}

\section{Subjects}

Fifteen patients with DSWPD who attended the neurology department of our hospital from June 2017 to October 2017 were selected as the observation group, and 15 sleep-normal individuals were selected as the control group. The study was approved by the ethics committee of The First Hospital of Hebei Medical University (No. 20190583). Simultaneously, the study was consistent with the Declaration of Helsinki.
Inclusion criteria: 1. Patients' age ranged from 16 to 60 years old; 2 . Subjective scale evaluation included the following: $\mathrm{PSQI}^{16} \leq 10$ points, $\mathrm{HAMA}_{14}{ }^{17}<7$ points, HAMD $_{24}{ }^{18} \leq 7$ points, ISI ${ }^{19} \leq 7$ points, MEQ $^{20}$ scores were $\leq 41$ points; 3 . Patients were able to wear a actigraphy for 2 weeks and record a sleep diary; in addition, all subjects met the diagnostic criteria for DSWPD in the ICSD-3. The inclusion criteria for the normal control group was MEQ score:42-58, and the rest of the criteria were the same as items 2 and 3 of the inclusion criteria for DSWPD patients.

Exclusion criteria: 1 . Patients aged $<16$ years or $>60$ years; 2. Patients with combined cognitive dysfunction with a Mini-Mental State Evaluation Scale (MMSE) ${ }^{21}$ score $>26$; 3. Patients with combined severe underlying diseases of the heart, respiratory system, or tumors; 4 . Patients with long-term bed rest, malnutrition, electrolyte disturbances, and anemia; 5. Patients who have been clearly diagnosed with other types of sleep disorders.

\section{Study Design and Conduct}

Age, sex, body mass index (BMI), and occupation were recorded separately for the 2 groups. All enrolled patients signed an informed consent form and were approved by the ethics committee. Both groups wore glasses (brand model PEGASI intelligent sleep glasses, PG16K02) that emitted approximately $470 \mathrm{~nm}$ blue light continuously for $1 \mathrm{~h}$ between 06:30 and 09:00 in the morning (1-2 $\mathrm{h}$ after waking up), limiting light exposure after 16:00 in the afternoon and avoiding bright light exposure at night, and instructing them to follow their natural resting time. The treatment was continued for 1 week. HAMA ${ }_{14}$, $\mathrm{HAMD}_{24}$, PSQI, ISI, ESS and MEQ scores were collected 1 week before and 1 week after the start of treatment in both groups Figure 1, and sleep diary and actigraphy sleep data were recorded at the same time. Among them, the sleep diary took the sleep latency (SL), total sleep time (TST), total time in bed (TTiB), sleep efficiency (SE), sleep quality (1 point for very poor, 2 points for poor or moderate, 3 points for good or very good, and 4 points for excellent), time of awakenings (TOA) and wakefulness after sleep onset (WASO), and average awakening time (AAT) as monitoring indexes.

\section{Statistical Analysis}

The frequency and composition ratios were used to describe the distribution of the count data; the quantitative data of the normal distribution were described using the 


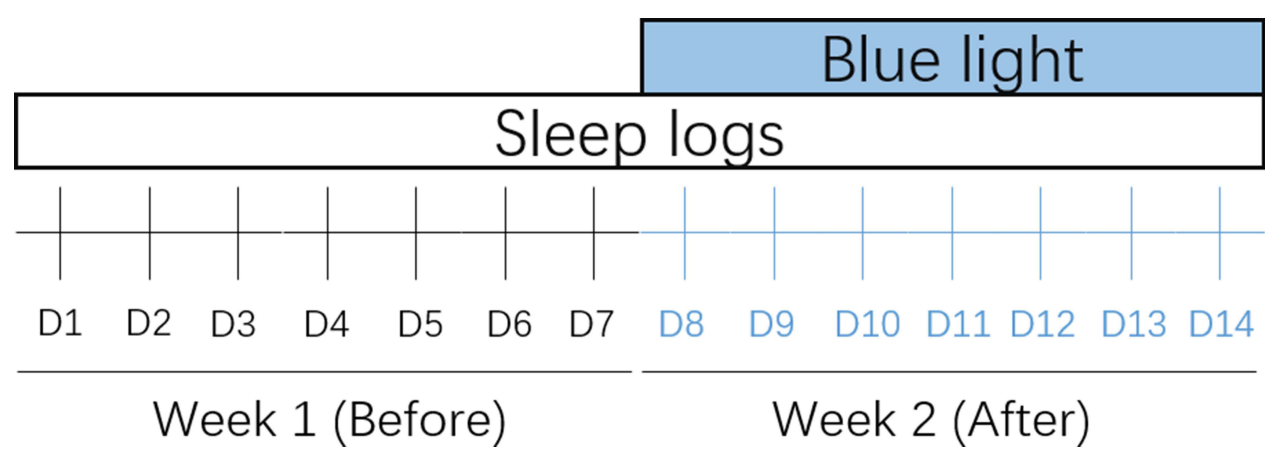

Figure I Schematic diagram study design. Sleep logs recording: Day I-7 in week I is before blue light treatment, Day 8-I4 in week 2 is during blue light treatment.

mean \pm standard deviation, and the paired $t$-test was used for the comparison before and after the test. The statistical test was a two-sided test with a test level of $p=0.05$. SPSS 21.0 software was used for statistical analysis.

\section{Results}

In this study, the age of the 15 DSWPD patients was 29.73 \pm 8.98 years, $26.75 \%$ were male, and their BMI was 22.72 $\pm 2.95 \mathrm{~kg} / \mathrm{m}^{2}$. The age of the 15 controls was $34.9 \pm 10.80$ years, $46.71 \%$ were male, and the BMI was $22.75 \pm 2.76 \mathrm{~kg}$ / $\mathrm{m}^{2}$. There was no statistically significant difference in the comparison of general information such as age, gender, $\mathrm{BMI}$, and occupation between the two groups $(\mathrm{P}>0.05)$.

\section{Changes in Sleep and Mood Before and After Blue Light Treatment Mood and Sleep Quality}

The total PSQI score $(3.50 \pm 2.03$ vs $3.00 \pm 1.97)$ was significantly lower before and after blue light treatment in the usual control group ( $\mathrm{P}=0.033)$; the PSQI-G score $(0.81 \pm 0.83$ vs 0.50 $\pm 0.63)$ was also significantly lower $(\mathrm{P}=0.025)$ Table 1 . DSWPD patients had significantly lower PSQI-G scores before and after blue light treatment $(\mathrm{P}=0.020)$. The MEQ scores of DSWPD patients increased significantly before and after the application of blue light therapy, which was statistically significant $(\mathrm{P}=0.002)$; the mean MEQ score of DSWPD patients shifted from 38.07 to 47.33 after treatment Table 2 .

\section{Sleep Diary}

The mean time spent in bed before and after blue light treatment increased in the control group, with a statistically significant difference $(P=0.018)$. Table 3 . Compared with before treatment, DSWPD patients had increased sleep quality scores after blue light treatment, with a statistically significant difference $(\mathrm{P}=0.033)$. On the other hand, TOA and WASO were significantly reduced after blue light treatment in DSWPD patients Table 4. The mean time to fall asleep $7 \mathrm{~d}$ after initiation was earlier than the mean time to fall asleep $7 \mathrm{~d}$ before treatment, and the mean sleep-wake phase of patients tended to shift somewhat forward. Figure 2A The mean time to sleep for patients was 11:19 (PM) for 7 consecutive $\mathrm{d}$ before treatment and 11:03 (PM) for 7 consecutive $\mathrm{d}$ after the start of treatment, and the mean time to sleep shifted by 16 min after treatment. Figure $2 \mathrm{~B}$

\section{Actigraphy}

Mean TTiB increased before and after blue light treatment in controls, with a statistically significant difference $(\mathrm{P}=$ 0.004) Table 5. No statistical difference in SL, SE, TTiB, TST, WASO, TOA and AAT data after blue light treatment in DSWPD patients Table 6.

Table I Comparison of Relative Data of Sleep Quality and Emotion Questionnaire Before and After Treatment in Control Group (Score, Mean士SD)

\begin{tabular}{|l|l|l|l|l|l|}
\hline & Before & After & Difference & $\boldsymbol{P}$ & $\boldsymbol{t}$ \\
\hline HAMA $_{14}$ & $1.94 \pm 1.77$ & $1.63 \pm 1.85$ & $-0.31 \pm 0.87$ & 0.166 & 2.049 \\
HAMD $_{24}$ & $1.38 \pm 1.46$ & $0.81 \pm 1.47$ & $-0.56 \pm 1.15$ & 0.071 & 3.809 \\
PSQI & $3.50 \pm 2.03$ & $3.00 \pm 1.97$ & $-0.50 \pm 0.82$ & 0.033 & 6.000 \\
PSQI-A & $0.81 \pm 0.54$ & $0.81 \pm 0.54$ & 0 & 1.000 & 0.000 \\
PSQI-B & $0.88 \pm 0.806$ & $0.69 \pm 0.79$ & $-0.19 \pm 0.40$ & 0.083 & 3.462 \\
PSQI-C & $0.38 \pm 0.50$ & $0.31 \pm 0.48$ & $-0.06 \pm 0.25$ & 0.317 & 1.000 \\
PSQI-D & $0.06 \pm 0.25$ & $0.13 \pm 0.34$ & $0.06 \pm 0.25$ & 0.317 & 1.000 \\
PSQI-E & $0.56 \pm 0.51$ & $0.56 \pm 0.51$ & 0 & 1.000 & \\
PSQI-F & 0 & 0 & 0 & 1.000 & \\
PSQI-G & $0.81 \pm 0.83$ & $0.50 \pm 0.63$ & $-0.31 \pm 1.25$ & 0.025 & 6.818 \\
ISI & $2.06 \pm 2.49$ & $1.50 \pm 2.13$ & $-0.56 \pm 1.41$ & 0.140 & 2.537 \\
ESS & $2.75 \pm 1.48$ & $3.06 \pm 1.88$ & $0.31 \pm 1.25$ & 0.380 & 1.000 \\
MEQ & $58.25 \pm 11.51$ & $57.56 \pm 10.71$ & $-0.69 \pm 4.06$ & 0.301 & 0.458 \\
\hline
\end{tabular}

Abbreviations: HAMAI4, Hamilton Anxiety Scale 14; HAMD24, Hamilton Depression Scale 24; PSQI, Pittsburgh sleep quality index; ISI, Insomnia severity index; ESS, Epworth Sleepiness Scale; MEQ, Morningness Eveningness Questionnaire. 
Table 2 Comparison of Relative Data of Sleep Quality and Emotion Questionnaire Before and After Treatment in Observation Group (Score, Mean \pm SD)

\begin{tabular}{|c|c|c|c|c|c|}
\hline & Before & After & Difference & $P$ & $t$ \\
\hline HAMA $_{14}$ & $2.33 \pm 1.68$ & $1.80 \pm 1.52$ & $0.53 \pm 1.25$ & 0.120 & 2.748 \\
\hline $\mathrm{HAMD}_{24}$ & $2.40 \pm 1.92$ & $2.27 \pm 1.10$ & $0.13 \pm 1.85$ & 1.000 & 0.078 \\
\hline PSQI & $6.47 \pm 1.77$ & $5.13 \pm 2.10$ & $1.33 \pm 2.74$ & 0.081 & 3.544 \\
\hline PSQI-A & $\mathrm{I} .40 \pm 0.5 \mathrm{I}$ & $1.20 \pm 0.68$ & $0.20 \pm 0.56$ & 0.180 & 1.909 \\
\hline PSQI-B & $1.87 \pm 0.99$ & $\mathrm{I} .40 \pm 0.9 \mathrm{I}$ & $0.47 \pm 0.92$ & 0.070 & 3.898 \\
\hline PSQI-C & $0.87 \pm 0.92$ & $0.67 \pm 0.62$ & $0.20 \pm 0.68$ & 0.257 & 1.313 \\
\hline PSQI-D & $0.60 \pm 0.51$ & $0.60 \pm 0.51$ & $0.00 \pm 0.65$ & 1.000 & 0.000 \\
\hline PSQI-E & $0.53 \pm 0.52$ & $0.07 \pm 0.26$ & $-0.07 \pm 0.59$ & 0.655 & 0.189 \\
\hline PSQI-F & 0 & $0.60 \pm 0.5 \mathrm{I}$ & $-0.07 \pm 0.26$ & 0.317 & 1.000 \\
\hline PSQI-G & $1.13 \pm 0.64$ & $0.07 \pm 0.26$ & $0.47 \pm 0.64$ & 0.020 & 7.977 \\
\hline ISI & $5.07 \pm 2.05$ & $4.33 \pm 2.58$ & $0.73 \pm 1.62$ & 0.102 & 3.058 \\
\hline ESS & $4.60 \pm 3.80$ & $3.47 \pm 1.41$ & $1.13 \pm 3.98$ & 0.324 & 1.216 \\
\hline MEQ & $38.07 \pm 9.00$ & $47.33 \pm 8.14$ & $-9.27 \pm 9.65$ & 0.002 & 13.840 \\
\hline
\end{tabular}

Abbreviations: HAMAI4, Hamilton Anxiety Scale I4; HAMD24, Hamilton Depression Scale 24; PSQI, Pittsburgh sleep quality index; ISI, Insomnia severity index; ESS, Epworth Sleepiness Scale; MEQ, Morningness Eveningness Questionnaire.

Table 3 Comparison of Sleep Diary Data Before and After Treatment in Normal Control Group (Mean \pm SD)

\begin{tabular}{|l|c|c|c|c|c|}
\hline & Before & After & Difference & $\boldsymbol{P}$ & $\boldsymbol{t}$ \\
\hline SL (min) & $21.45 \pm 19.15$ & $17.52 \pm 8.99$ & $-3.926 \pm 14.35$ & 0.291 & 1.198 \\
TST (min) & $442.50 \pm 43.07$ & $451.31 \pm 48.64$ & $8.82 \pm 45.12$ & 0.446 & 0.611 \\
TTiB (min) & $432.99 \pm 24.45$ & $465.92 \pm 36.05$ & $32.93 \pm 13.92$ & 0.018 & 22.380 \\
SE (\%) & $92.76 \pm 5.44$ & $93.78 \pm 4.39$ & $1.018 \pm 1.80$ & 0.577 & 1.422 \\
SQ (score) & $2.65 \pm 0.38$ & $2.76 \pm 0.39$ & $0.09 \pm 0.27$ & 0.248 & 1.452 \\
TOA (time) & $1.34 \pm 0.89$ & $1.13 \pm 1.02$ & $-0.21 \pm 0.67$ & 0.241 & 1.488 \\
WASO (min) & $9.46 \pm 6.14$ & $8.33 \pm 6.67$ & $-1.13 \pm 5.14$ & 0.392 & 0.778 \\
\hline
\end{tabular}

Abbreviations: SL, Sleep Time; TST, Total Sleep Time; TTiB, Total Time in Bed; SE, Sleep efficiency percent; SQ, Sleep Quality; TOA, Total Arousal Time; WASO, Wake After Sleep Onset.

Table 4 Comparison of Sleep Diary Data Before and After Treatment in Observation Group (Mean \pm SD)

\begin{tabular}{|l|c|c|c|c|c|}
\hline & Before & After & Difference & $P$ & t \\
\hline SL (min) & $33.36 \pm 25.47$ & $21.37(11.91)$ & $11.98 \pm 24.92$ & 0.084 & 3.469 \\
TST (mim) & $416.95 \pm 39.65$ & $426.56 \pm 67.07$ & $-9.61 \pm 57.61$ & 0.529 & 0.418 \\
TTiB (min) & $480.04 \pm 25.93$ & $477.68 \pm 38.14$ & $2.36 \pm 37.29$ & 0.810 & 0.060 \\
SE (\%) & $86.85 \pm 6.68$ & $88.85 \pm 9.34$ & $2.00 \pm 2.96$ & 0.504 & 0.823 \\
SQ (score) & $2.47 \pm 0.45$ & $2.78 \pm 0.33$ & $-0.31 \pm 0.46$ & 0.033 & 6.839 \\
TOA (time) & $0.85 \pm 0.65$ & $0.61 \pm 0.72$ & $0.25 \pm 0.39$ & 0.028 & 5.975 \\
WASO (min) & $9.39 \pm 8.44$ & $6.00 \pm 7.17$ & $3.39 \pm 6.05$ & 0.044 & 4.705 \\
\hline
\end{tabular}

Abbreviations: SL, Sleep Time; TST, Total Sleep Time; TTiB, Total Time in Bed, SE, Sleep efficiency percent; SQ, Sleep Quality; TOA, Total Arousal Time; WASO, Wake After Sleep Onset.

Correlation Analysis Between the Efficacy of Patients with Delayed Sleep-Wake Phase Disorder and Sleep Indicators

By Pearson correlation analysis, there were significant correlations between the efficacy of patients with sleep-wake phase delay disorder and HAMA14, HAMD24, PSQI, ISI, ESS, MEQ, SL, TST, TTiB, sleep quality, TOA, WASO, AAT, and SE (r=0.183, 0.243, 0.183, 0.137, 0.321, -0.203, $0.198,0.167,0.317,0.278,0.196,0.287,0.298,0.149$, all $\mathrm{p}<0.001)$. 


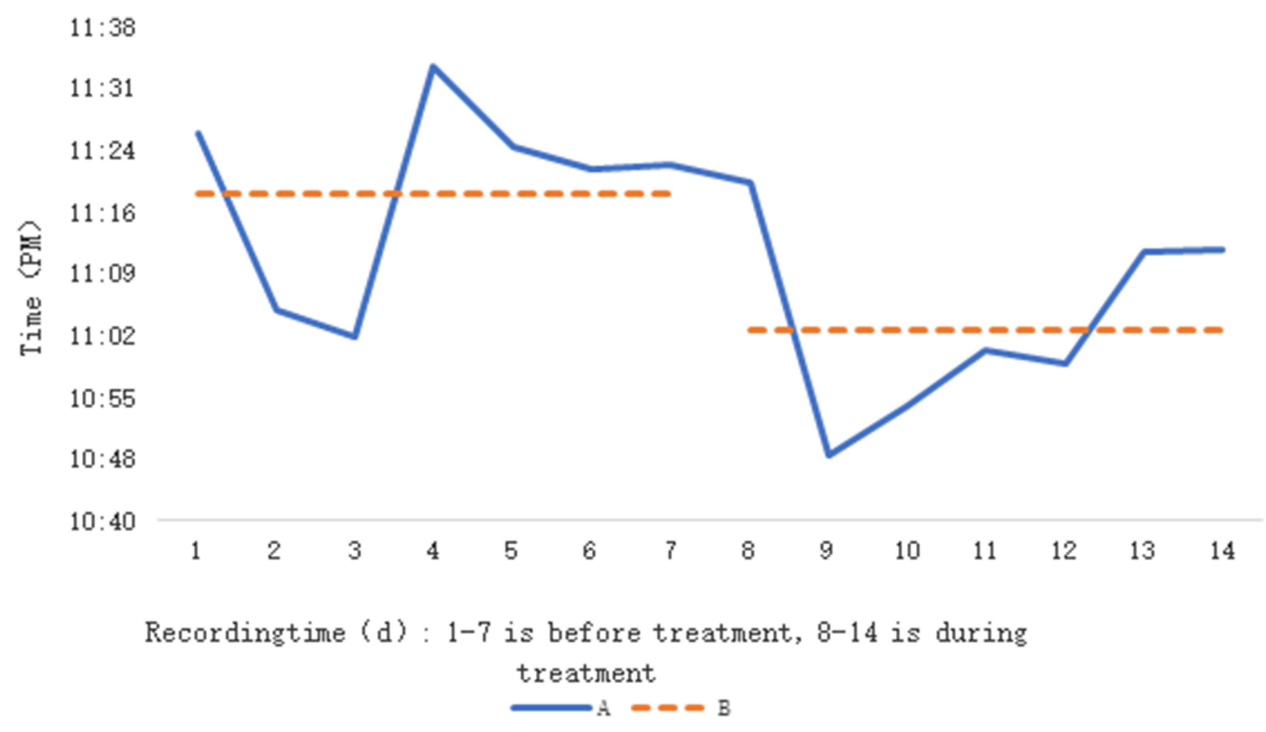

Figure 2 Average sleeping time of patients with DSWPD before and after blue light treatment were compared; (A) linear trend of average sleeping time before and after treatment; (B) average sleeping time before (11:19) and after (11:03) treatment.

\section{Multifactorial Logistic Regression Analysis of} Factors Influencing the Efficacy of Patients with Sleep-Wake Phase Delay Disorder

After correcting for age, gender, BMI, and occupation, a multifactorial logistic regression analysis was performed with clinical efficacy as the dependent variable (1=ineffective, 0=effective), and HAMA14, HAMD24, PSQI, ISI, ESS, MEQ, SL, TST, TTiB, SQ, TOA, WASO, AAT, and SE as independent variables, and found that The factors influencing the efficacy of patients with sleep-wake phase delay disorder were PSQI, ISI, ESS, MEQ, SL, TST, TTiB, sleep quality, TOA, WASO, AAT, and SE, as shown in Table 7.

Table 5 Comparison of Relative Data of Actigraphy Before and After Treatment in Control Group (Mean \pm SD)

\begin{tabular}{|l|c|c|c|c|c|}
\hline & Before & After & Difference & $\boldsymbol{P}$ & $\boldsymbol{t}$ \\
\hline SL (min) & $4.37 \pm 3.39$ & $6.75 \pm 5.31$ & $2.38 \pm 6.40$ & 0.158 & 2.204 \\
SE (min) & $84.64 \pm 6.22$ & $83.14 \pm 5.99$ & $-1.499 \pm 6.66$ & 0.569 & 0.807 \\
TTiB (min) & $464.88 \pm 32.17$ & $482.30 \pm 36.99$ & $17.41 \pm 20.23$ & 0.004 & $11.85 \mathrm{I}$ \\
TST (min) & $392.77 \pm 34.02$ & $402.00 \pm 51.67$ & $9.23 \pm 30.01$ & 0.238 & 1.512 \\
WAS (min) & $67.72 \pm 28.42$ & $73.54 \pm 23.61$ & $5.82 \pm 30.16$ & 0.569 & 0.597 \\
TOA (time) & $22.69 \pm 8.41$ & $22.72 \pm 5.63$ & $0.03 \pm 7.62$ & 0.605 & 0.000 \\
AAT (min) & $2.89 \pm 0.53$ & $3.22 \pm 0.69$ & $0.32 \pm 0.82$ & 0.313 & 2.460 \\
\hline
\end{tabular}

Abbreviations: SL, Sleep Time; SE, Sleep efficiency percent; TTiB, Total Time in Bed; TST, Total Sleep Time; WASO, Wake After Sleep Onset; TOA, Total Arousal Time; AAT, Average Arousal Time.

Table 6 Comparison of Relative Data of Actigraphy Before and After Treatment in Observation Group (Mean \pm SD)

\begin{tabular}{|l|c|c|c|c|c|}
\hline & Before & \multicolumn{1}{|l|}{ After } & Difference & $\boldsymbol{P}$ & $\boldsymbol{t}$ \\
\hline SL (min) & $6.29 \pm 5.21$ & $5.69 \pm 5.00$ & $0.82 \pm 7.95$ & 0.707 & 0.148 \\
SE (min) & $85.44 \pm 5.73$ & $84.33 \pm 6.83$ & $1.27 \pm 9.59$ & 0.778 & 0.247 \\
TTiB (min) & $465.86 \pm 44.59$ & $462.94 \pm 50.14$ & $2.62 \pm 29.01$ & 0.741 & 0.114 \\
TST (min) & $397.80 \pm 44.91$ & $389.86 \pm 48.40$ & $8.38 \pm 59.95$ & 0.925 & 0.274 \\
WASO (min) & $58.55 \pm 25.30$ & $67.39 \pm 33.19$ & $-9.35 \pm 43.19$ & 0.807 & 0.610 \\
TOA (time) & $20.87 \pm 9.48$ & $20.82 \pm 6.65$ & $0.16 \pm 9.43$ & 0.330 & 0.004 \\
AAT (min) & $2.86 \pm 0.78$ & $3.18 \pm 0.86$ & $-0.39 \pm 1.25$ & 0.261 & \\
\hline
\end{tabular}

Abbreviations: SL, Sleep Time; SE, Sleep efficiency percent; TTiB, Total Time in Bed; TST, Total Sleep Time; WASO, Wake After Sleep Onset; TOA, Total Arousal Time; AAT, Average Arousal Time. 
Table 7 Multifactorial Logistic Regression Analysis of Factors Influencing the Efficacy of Patients with Sleep-Wake Phase Delay Disorder

\begin{tabular}{|c|c|c|c|c|c|}
\hline & $\begin{array}{l}\text { Regression } \\
\text { Coefficient }\end{array}$ & Standard Error & Wald $\chi^{2}$ & $\mathbf{P}$ & OR value $(95 \% \mathrm{Cl})$ \\
\hline HAMA $_{14}$ & 0.091 & 0.023 & 0.812 & 0.623 & I.102 (0.922-I.45I) \\
\hline $\mathrm{HAMD}_{24}$ & 0.783 & $0.41 \mathrm{I}$ & 3.812 & 0.061 & $2.179(0.992-4.233)$ \\
\hline PSQI & 1.738 & 0.492 & 13.192 & $<0.001$ & $5.212(2.091-12.133)$ \\
\hline ISI & 0.103 & 0.313 & 9.712 & $<0.001$ & $3.502(3.102-4.115)$ \\
\hline ESS & 1.047 & 0.158 & 17.568 & $<0.001$ & $2.762(1.698-4.283)$ \\
\hline MEQ & -0.44 I & 0.173 & 4.192 & 0.015 & $1.712(1.031-2.109)$ \\
\hline SL & 1.042 & 0.282 & 4.383 & 0.023 & $2.354(1.538-5.879)$ \\
\hline TST & $\mathrm{I} .887$ & 0.116 & 4.193 & $<0.001$ & $6.928(2.012-19.873)$ \\
\hline TTiB & 1.803 & 0.862 & 14.393 & $<0.001$ & $5.672(4.092-11.289)$ \\
\hline SQ & 1.703 & 0.579 & 8.319 & 0.004 & $5.765(1.987-17.654)$ \\
\hline TOA & 0.478 & 0.159 & 10.298 & $<0.001$ & 1.791 (1.209-2.213) \\
\hline WASO & 1.276 & 0.332 & 12.187 & $<0.001$ & $3.287(1.765-5.982)$ \\
\hline AAT & 2.065 & 0.768 & 6.876 & 0.007 & 7.917 (1.689-34.292) \\
\hline SE & 1.762 & 0.463 & 14.287 & $<0.001$ & $5.387(2.176-12.098)$ \\
\hline
\end{tabular}

Abbreviations: HAMAI4, Hamilton Anxiety Scale I4; HAMD24, Hamilton Depression Scale 24; PSQI, Pittsburgh sleep quality index; ISI, Insomnia severity index; ESS, Epworth Sleepiness Scale; MEQ, Morningness Eveningness Questionnaire; SL, Sleep Time; TTiB, Total Time in Bed; SQ, Sleep Quality; TST, Total Sleep Time; WASO, Wake After Sleep Onset; TOA, Total Arousal Time; AAT, Average Arousal Time; SE, Sleep efficiency percent.

\section{The Experience of Participants with Blue Light Glasses}

$77.6 \%(23 / 30)$ of the subjects were willing to continue using blue light glasses in the future, $16.7 \%(5 / 30)$ were neutral, and $6.7 \%(2 / 30)$ did not want to continue using them because they did not benefit significantly from their use. 90\% (27/30) of the patients thought that blue light glasses were light to wear, easy to operate, and could be learned quickly. $23.3 \%$ (7/30) of the patients felt that the comfort of the glasses was poor.

\section{Discussion}

Although the pathogenesis of DSWPD is uncertain, there is an accumulation of evidence that the human biological clock regulation center is located in the suprachiasmatic nuclei (SCN) of the anterior hypothalamus. ${ }^{22}$ The SCN contains autonomous oscillating cells with a cycle of about 24 hours, and these cells sense the shift of the external circadian light environment by receiving optic nerve impulses from the retina, and maintain their own circadian rhythm synchronized with the natural cycle, known as the "circadian pacemaker". ${ }^{23}$ Light is the strongest synchronizer of human circadian rhythms, with morning light exposure advancing circadian rhythms and nighttime light exposure delaying them. ${ }^{24,25}$ Previous studies have found that DSWPD patients have more light exposure at night (22:00 pm to 2:00 am) and less light exposure in the morning (8:00 to 9:00 am and 10:00 to 12:00 am) compared to normal sleepers, ${ }^{26}$ and this is how light therapy works. It has been reported that giving DSWPD patients light at an intensity of 2000-2500 LUX in the morning (6:00-9:00 am) while limiting their bright light exposure at night shifted their sleepwake phase forward. ${ }^{9,24}$ Further studies found that light in the wavelength range of $420 \mathrm{~nm}$ to $500 \mathrm{~nm}$ has a significant effect on the circadian rhythm of the human body, and that short wavelength light near 460nm (blue light) has the strongest inhibitory effect on endogenous melatonin secretion at the same light intensity, and also has the most significant effect on the phase change of biological clock, wakefulness and pupil light reflection, which is called the "action spectrum". 14,15,27 However, the specific light duration, intensity and spectrum are not elaborated, and there are no such therapeutic products in China, nor are there any reports of clinical application.

In this study, after excluding the effects of drugs and behavioral therapies, the beneficial blue light with a wavelength of $470 \mathrm{~nm}$ was used, which did not cause visual damage to the subjects, mainly because the exposure time to blue light was very short, which was not enough to cause any damage to the eyes. ${ }^{28}$ In the other hand, blue light had a more pronounced effect on the body's circadian rhythm. ${ }^{9}$ In our study, DSWPD patients and controls in the study were treated with $470 \mathrm{~nm}$ blue light glasses for $1 \mathrm{~h}$ in the morning between 06:30 and 09:00 am, respectively, and light was restricted after 16:00 pm and bright light was avoided at night. After 1 week of treatment, subjects in both groups showed benefits in subjective nighttime sleep quality and daytime function the 
following day. After blue light treatment, DWSPD patients had fewer nocturnal awakenings and shorter awakening time, and changed from "moderate night type" before treatment to "intermediate type" after treatment; the mean time to sleep shifted 16 min after $7 \mathrm{~d}$ of treatment compared with that before treatment, suggesting that the sleep-wake phase of the patients was shifted forward to a certain extent. These findings are consistent with this study that morning blue light therapy can support a sleep advancement program by phasing in circadian rhythms as well as sleep duration. $^{29}$

In addition to the side effects of conventional therapy, the light treatment process restricts the patient to a limited space for a certain period of time, and this restriction further leads to poor patient acceptance. ${ }^{30}$ In this survey, most of the subjects showed that they could receive blue light glasses because the glasses were easy to carry and hardly interfered with their regular life. In addition, patients wearing blue light glasses hardly noticed side effects such as eye fatigue, nausea, agitation, mania and migraine. In general, patients have a positive attitude towards blue light glasses and are willing to continue using them.

Light therapy has been used abroad for the treatment of sleep disorders, dementia, dermatological disorders, mental and emotional disorders, and the therapeutic effects have been confirmed by research. ${ }^{31-34}$ However, the research and use of light therapy in China is still relatively small, especially for the clinical application of sleep-wake phase disorder diseases which needs further development and in-depth research. There are some limitations in this study. The study sample was very small because the patients were recruited from one hospital. In addition, this study covered only a short 2-week trial period, and it remains unclear whether blue light has long-term efficacy in the treatment of patients with DSWPD. Furthermore, if some patients wearing glasses wake up earlier in the morning, leading to the possibility that they are exposed to more morning light, this may affect the advancement of the phase, and it is difficult to distinguish the effect between blue glasses or morning light. These issues need to be considered as much as possible in further studies.

In summary, wearing blue light glasses can effectively treat DSWPD patients. During the two weeks of treatment, no adverse effects of blue light were observed. Blue light therapy improved the subjective sleep quality, reduced the number and duration of night awakenings, and showed higher acceptance of blue light glasses for DSWPD. Together, these results demonstrate that blue light glasses are clinically feasible and have good user compliance and acceptance as a new type of light therapy.

\section{Data Sharing Statement}

The datasets generated and analyzed during the current study are available from the corresponding author on reasonable request.

\section{Ethics Approval and Consent to Participate}

The study protocol was approved by the Ethics Committee of the First Hospital of Hebei Medical University. Informed consent was obtained from all the study subjects before enrollment.

\section{Author Contributions}

All authors made a significant contribution to the work reported, whether that is in the conception, study design, execution, acquisition of data, analysis and interpretation, or in all these areas; took part in drafting, revising or critically reviewing the article; gave final approval of the version to be published; have agreed on the journal to which the article has been submitted; and have agreed on the journal to which the article has been submitted.

\section{Funding}

This study was supported by Scientific Research Fund Project of Hebei Provincial Health Commission (No. 20190460 and 20180221).

\section{Disclosure}

The authors declare that they have no competing interests.

\section{References}

1. Thorpy M. International classification of sleep disorders. In: Sleep Disorders Medicine. Springer; 2017:475-484.

2. Nesbitt AD. Delayed sleep-wake phase disorder. $J$ Thorac Dis. 2018;10(Suppl 1):S103. doi:10.21037/jtd.2018.01.11

3. Murray JM, Sletten TL, Magee M, et al. Prevalence of circadian misalignment and its association with depressive symptoms in delayed sleep phase disorder. Sleep. 2017;40:1.

4. Richardson C, Cain N, Bartel K, et al. A randomised controlled trial of bright light therapy and morning activity for adolescents and young adults with delayed sleep-wake phase disorder. Sleep Med. 2018;45:114-123. doi:10.1016/j.sleep.2018.02.001

5. Sivertsen B, Harvey AG, Gradisar M, et al. Delayed sleep-wake phase disorder in young adults: prevalence and correlates from a national survey of Norwegian university students. Sleep Med. 2021;77:184-191. doi:10.1016/j.sleep.2020.09.028

6. Richardson CE, Gradisar M, Barbero SC. Are cognitive "insomnia" processes involved in the development and maintenance of delayed sleep wake phase disorder? Sleep Med Rev. 2016;26:1-8. doi:10.1016/ j.smrv.2015.05.001

7. Archer SN, Carpen JD, Gibson M, et al. Polymorphism in the PER3 promoter associates with diurnal preference and delayed sleep phase disorder. Sleep. 2010;33(5):695-701. doi:10.1093/sleep/33.5.695 
8. Richardson C, Gradisar M. Depressed mood and repetitive negative thinking in delayed sleep-wake phase disorder: treatment effects and a comparison with good sleepers. J Sleep Res. 2021;e13452. doi:10.1111/jsr.13452

9. Magee M, Marbas EM, Wright KP, et al. Diagnosis, cause, and treatment approaches for delayed sleep-wake phase disorder. Sleep Med Clin. 2016;11(3):389-401. doi:10.1016/j.jsmc.2016.05.004

10. Liu J, Clough SJ, Hutchinson AJ, et al. MT1 and MT2 melatonin receptors: a therapeutic perspective. Annu Rev Pharmacol Toxicol. 2016;56:361-383. doi:10.1146/annurev-pharmtox-010814-124742

11. Lau T, Lovato N, Lack L. Evaluation of a portable light device for phase advancing the circadian rhythm in the home environment. Sleep Biol Rhythms. 2018;16(4):405-411. doi:10.1007/s41105-0180167-5

12. Kogan AO, Guilford PM. Side effects of short-term 10,000-lux light therapy. Am J Psychiatry. 1998;155(2):293-294.

13. Zerbini G, Kantermann T, Merrow MJ. Strategies to decrease social jetlag: reducing evening blue light advances sleep and melatonin. Eur $J$ Neurosci. 2020;51(12):2355-2366. doi:10.1111/ejn.14293

14. Videnovic A, Willis GL. Circadian system - a novel diagnostic and therapeutic target in Parkinson's disease? Mov Disorders. 2016;31 (3):260-269. doi:10.1002/mds.26509

15. Boutrel B, Koob GF. What keeps us awake: the neuropharmacology of stimulants and wakefulness promoting medications. Sleep. 2004;27(6):1181-1194. doi:10.1093/sleep/27.6.1181

16. Carpenter JS, Andrykowski MA. Psychometric evaluation of the Pittsburgh sleep quality index. J Psychosom Res. 1998;45(1):5-13. doi:10.1016/S0022-3999(97)00298-5

17. Maier W, Buller R, Philipp M, et al. The Hamilton anxiety scale: reliability, validity and sensitivity to change in anxiety and depressive disorders. J Affect Disord. 1988;14(1):61-68. doi:10.1016/01650327(88)90072-9

18. Williams JB. A structured interview guide for the Hamilton depression rating scale. Arch Gen Psychiatry. 1988;45(8):742-747.

19. Bastien $\mathrm{CH}$, Vallières A, Morin CM. Validation of the Insomnia Severity Index as an outcome measure for insomnia research. Sleep Med. 2001;2(4):297-307.

20. Carciofo R, Du F, Song N, et al. Age-related chronotype differences in Chinese, and reliability assessment of a reduced version of the Chinese Morningness Eveningness Questionnaire. Sleep Biol Rhythms. 2012;10(4):310-318.

21. Kukull W, Larson E, Teri L, et al. The mini-mental state examination score and the clinical diagnosis of dementia. J Clin Epidemiol. 1994;47(9):1061-1067. doi:10.1016/0895-4356(94)90122-8

22. Landgraf D, Koch CE, Oster H. Embryonic development of circadian clocks in the mammalian suprachiasmatic nuclei. Front Neuroanat. 2014;8:143. doi:10.3389/fnana.2014.00143

23. Turner PL, Mainster MA. Circadian photoreception: ageing and the eye's important role in systemic health. Br J Ophthalmol. 2008;92 (11):1439-1444. doi:10.1136/bjo.2008.141747
24. Saxvig IW, Wilhelmsen-Langeland A, Pallesen S, et al. A randomized controlled trial with bright light and melatonin for delayed sleep phase disorder: effects on subjective and objective sleep. Chronobiol Int. 2014;31(1):72-86. doi:10.3109/ 07420528.2013.823200

25. Berson DM, Dunn FA, Takao M. Phototransduction by retinal ganglion cells that set the circadian clock. Science. 2002;295 (5557):1070-1073. doi:10.1126/science.1067262

26. Wilson IV J, Reid KJ, Braun RI, et al. Habitual light exposure relative to circadian timing in delayed sleep-wake phase disorder. Sleep. 2018;41(11):zsy166. doi:10.1093/sleep/zsy166

27. Cajochen C, Jud C, Münch M, et al. Evening exposure to blue light stimulates the expression of the clock gene PER2 in humans. Eur $J$ Neurosci. 2006;23(4):1082-1086. doi:10.1111/j.1460-9568.20 06.04613.x

28. Tosini G, Ferguson I, Tsubota K. Effects of blue light on the circadian system and eye physiology. Mol Vision. 2016;22:61.

29. Geerdink M, Walbeek TJ, Beersma DG, et al. Short blue light pulses (30 $\mathrm{min}$ ) in the morning support a sleep-advancing protocol in a home setting. J Biol Rhythms. 2016;31(5):483-497. doi:10.1177/ 0748730416657462

30. Auger RR, Burgess HJ, Emens JS, et al. Clinical practice guideline for the treatment of intrinsic circadian rhythm sleep-wake disorders: advanced sleep-wake phase disorder (ASWPD), delayed sleep-wake phase disorder (DSWPD), non-24-hour sleep-wake rhythm disorder (N24SWD), and irregular sleep-wake rhythm disorder (ISWRD). An update for 2015: an American Academy of Sleep Medicine clinical practice guideline. J Clin Sleep Med. 2015;11(10):1199-1236. doi:10.5664/jcsm.5100

31. Janků K, Šmotek M, Fárková E, et al. Block the light and sleep well: evening blue light filtration as a part of cognitive behavioral therapy for insomnia. Chronobiol Int. 2020;37(2):248-259. doi:10.1080/ 07420528.2019.1692859

32. Faulkner SM, Bee PE, Meyer N, et al. Light therapies to improve sleep in intrinsic circadian rhythm sleep disorders and neuro-psychiatric illness: a systematic review and meta-analysis. Sleep Med Rev. 2019;46:108-123. doi:10.1016/j.smrv.2019.04.012

33. Sorbellini E, Rucco M, Rinaldi F. Photodynamic and photobiological effects of light-emitting diode (LED) therapy in dermatological disease: an update. Lasers Med Sci. 2018;33(7):1431-1439. doi:10.1007/s10103-018-2584-8

34. Esaki Y, Kitajima T, Ito Y, et al. Wearing blue light-blocking glasses in the evening advances circadian rhythms in the patients with delayed sleep phase disorder: an open-label trial. Chronobiol Int. 2016;33(8):1037-1044. doi:10.1080/07420528.2016.1194289
Nature and Science of Sleep

\section{Publish your work in this journal}

Nature and Science of Sleep is an international, peer-reviewed, open access journal covering all aspects of sleep science and sleep medicine, including the neurophysiology and functions of sleep, the genetics of sleep, sleep and society, biological rhythms, dreaming, sleep disorders and therapy, and strategies to optimize healthy sleep.
The manuscript management system is completely online and includes a very quick and fair peer-review system, which is all easy to use. Visit http://www.dovepress.com/testimonials.php to read real quotes from published authors. 